\title{
Suspension rheology under oscillatory shear and its geophysical implications
}

\author{
Ikuro Sumita $^{\mathrm{a}, *}$, Michael Manga ${ }^{\mathrm{b}}$ \\ a Division of Earth and Environmental Sciences, Graduate School of Natural Sciences and Technology, Kanazawa University, Kanazawa, 920-1192, Japan \\ b Department of Earth and Planetary Science, University of California, Berkeley, 307 McCone Hall, Berkeley CA, 94720-4767 USA
}

\section{A R T I C L E I N F O}

\section{Article history:}

Received 24 July 2007

Received in revised form 27 February 2008

Accepted 29 February 2008

Available online 14 March 2008

Editor: C.P. Jaupart

\section{Keywords:}

suspension

rheology

coseismic response

liquefaction

dilatancy

triggering

\begin{abstract}
A B S T R A C T
Dynamic strain generated by seismic waves is often invoked to explain coseismic responses to earthquakes, such as liquefaction, dilatancy and the remote triggering of earthquakes and eruptions. In order to expand our understanding of the conditions under which such responses occur, we measure the rheology of non-Brownian particle suspensions under oscillatory shear at frequencies corresponding to the seismic band $(0.1-10 \mathrm{~Hz})$. We characterize the changes in rheology as a function of volumetric particle packing fraction (0.2-0.6), particle diameter $(9,39 \mu \mathrm{m})$, and the viscosity of the suspending fluid $(0.97,12.14$ Pas). By varying the stress (corresponding to a strain range $10^{-5}<\gamma<10^{1}$ ) we identify three rheological regimes. In order of increasing strain amplitude these are (I) linear viscoelastic, (II) shear-thinning and (III) shear-thickening. Transitions between regimes are better defined by a critical strain, rather than stress, particularly for large packing fractions. We find that critical strains are independent of the shearing frequency and fluid viscosity, whereas they decrease with particle packing fraction. We also monitor how the rheology recovers after imposing a large amplitude shear and find that the time scale for recovery is shorter for larger particles, suggesting that structural recovery is controlled by permeable fluid flow. Our results are consistent with the different regimes identified in previous shear tests of water-saturated sands which have a smaller fluid viscosity, suggesting that the same strain criteria can be applied to a wide variety of geological situations.
\end{abstract}

(c) 2008 Elsevier B.V. All rights reserved.

\section{Introduction}

Dense suspensions of solid particles in viscous fluids are common in geology; some examples include water or oil saturated sediments, muds, and crystal-bearing magma. At rest, these dense suspensions are solid-like, and do not flow owing to the friction between particles that are in contact. However, when shaken or sheared at sufficiently large amplitudes these materials start to flow. For still larger shaking, dilatancy occurs owing to particle displacement becoming so large such that the inter-particle pore space increases. Here dilatancy refers to the volumetric increase of the solid-liquid composite caused by the structural change of the solid particle network. In geological situations, such shaking or shearing can be caused by seismic waves, and is responsible for phenomena such as liquefaction and changes in well water level or stream flow (for a review see Montgomery and Manga, 2003). It has also been proposed that liquefaction can be responsible for eruption of mud volcanoes (Manga and Brodsky, 2006) as well as triggered eruption of volcanoes (Hill et al., 2002). Crystal-bearing magma is quite different from water-saturated sands, yet they do share common rheology. For example, the effect of dilatancy has been incorporated in the recent models of magma rheology (Koenders and Petford, 2000; Petford and Koenders, 2003).

\footnotetext{
* Corresponding author. Fax: +81 762646545.

E-mail address: sumita@hakusan.s.kanazawa-u.ac.jp (I. Sumita).
}

In order to understand the conditions needed to initiate liquefaction and dilatancy, rheology measurement of geological suspensions under oscillatory shear at seismic frequencies $(\sim 1 \mathrm{~Hz})$, with variable strain (or stress) amplitude is needed. These conditions can be influenced by a number of parameters such as the particle packing fraction $(\phi)$, particle diameter and liquid viscosity, as well as the shearing amplitude and frequency. Many studies in geotechnical engineering have assessed the susceptibility of a particular sand or soil to liquefaction at small strains (see Vucetic (1994) for a review) and dilatancy at large strains (e.g., Hatanaka et al., 1997). However these measurements were limited to water-saturated cases, and it is uncertain whether the same conditions apply to suspensions in more viscous liquids, which is relevant to magmatic suspensions as well as the case in which the water suspends fine particles which increases the effective viscosity (Iverson, 1997). Oscillatory shear measurements of near-solidus to partially-molten rocks have also been made, but are limited to small strains (e.g., Bagdassarov et al., 1994; James et al., 2004; Fontaine et al., 2005).

As a complimentary approach, rather than using real geological suspensions, we can use analogue materials and study their rheology using rheometers. In this paper we adopt this approach because we wish to constrain the factors which determine the conditions needed for the rheological changes. Although the experiments are in many ways simplified, they capture the same physics encountered in geological situations. For example, we use particles with sufficiently large diameters such that the effect of Brownian motion is negligible, 
yet still in the regime where inertia is also negligible. This is a condition similar to most geological situations, as we show in Section 2 using dimensionless numbers.

For an analogue material we use polystyrene beads in a silicone oil, which we consider as the simplest analogue material of crystalbearing magmatic suspensions and water-saturated sands and soils. Such analogue material is much simplified (uniform spherical particles, fully fluid saturated) compared to geological suspensions, but allows us to independently change the aforementioned parameters. For example, using nearly neutrally buoyant particles allow us to vary the particle packing fraction from dilute $(\phi=0.2)$ to dense $(\phi=0.6)$.

For rheology measurements we use high-precision rheometer which can deform samples under oscillatory shear at wide frequency range, $0.01-100 \mathrm{~Hz}$ and to measure the resulting strains over a wide range of $10^{-5}$ to 10 . These ranges are the same as the typical range of frequency and strain that accompanies seismic shear waves, thus allowing us to conduct analogue liquefaction and dilatancy experiments. Varying imposed frequency is important when we wish to argue whether ground displacement (strain), velocity or acceleration is best suited to define the onset of the rheological changes. It is known that particle suspensions have linear viscoelastic properties when they are sheared under small amplitudes, but when they are sheared under larger amplitudes, they show non-linear viscoelasticity whose properties depend on strain amplitude. However, it is only recently that suspension rheology under very large amplitude oscillatory shear has begun to be explored (Heymann et al., 2002; Narumi et al., 2005). Our experiments are designed to extend these works for the purpose of understanding the condition and the factors which determine the onset of liquefaction and dilatancy.

\section{Experimental methods}

Rheology measurements are made using a HAAKE Rheoscope with a truncated cone and plate geometry (Fig. 1a. For most measurements, we use a cone spindle with a smooth surface that has a diameter of $60 \mathrm{~mm}$, a cone angle of $4^{\circ}$, and a minimum gap width of $0.140 \mathrm{~mm}$. For the measurements shown later in Fig. 7, we use a cone with a diameter of

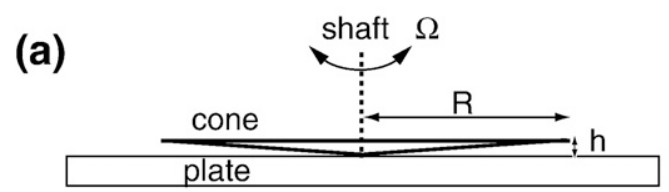

(b)

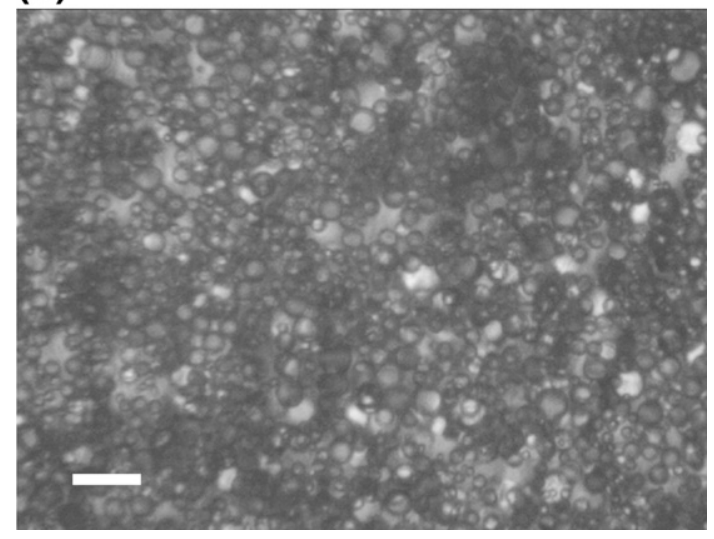

Fig. 1. (a) A schematic diagram showing the cone and plate geometry of the rheometer. The cone radius is $R=30 \mathrm{~mm}$ and the cone slope angle is $4^{\circ}$. The sample is sandwiched between the cone and plate and is deformed under an oscillatory shear and the resulting angular displacement $\Omega$ is measured to obtain strain. (b) A photograph of the particle suspensions under the Rheoscope. A scale bar is $50 \mu \mathrm{m}$. Here, the particle diameter is $9 \mu \mathrm{m}$, packing fraction is 0.5 .
$70 \mathrm{~mm}$ and a cone angle of $1^{\circ}$. We find no systematic difference between the measured values obtained with different cone spindles. For the solid particles, we use spherical polystyrene particles (Techpolymer SBX series, Sekisui Plastics Co., Japan) with a particle diameter of $8.6 \pm 3.1 \mu \mathrm{m}$ and $38.8 \pm 13.6 \mu \mathrm{m}$, and a density of $1060 \mathrm{~kg} \mathrm{~m}^{-3}$. For the suspending fluid, we use silicone fluids (Dow Corning 200) with viscosities of 0.97 and 12.14 Pas and densities of 970 and $971 \mathrm{~kg} \mathrm{~m}^{-3}$, respectively. Samples with packing fractions varying from 0.2 to 0.6 are prepared by thoroughly mixing the particles and the silicone fluid to form a uniform paste. Here packing fraction is defined as the volumetric fraction occupied by the particles. A photograph of a sample in the Rheoscope is shown in Fig. 1b. A thermostat controlled fluid with a fixed temperature of $24{ }^{\circ} \mathrm{C}$ is circulated through the base plate of the rheometer and all measurements were made at this temperature.

The typical experimental procedure is as follows. We first preshear the sample under a large oscillatory strain amplitude of $\gamma=10$ and frequency of $1 \mathrm{~Hz}$ for 20-30 s to reset the initial anomalous stress (Heymann et al., 2002). We then impose a periodic shear stress $\sigma=\sigma_{0}$ $\sin 2 \pi f t$, and measure the complex shear modulus $G^{*}\left(f, \sigma_{0}\right)$

$G^{*}\left(f, \sigma_{0}\right)=G^{\prime}+i G^{\prime \prime}=\left|G^{*}\right| \cos \theta+i\left|G^{*}\right| \sin \theta$

under a fixed frequency $f$ and stress amplitude $\sigma_{0}$, and measure the resulting strain and phase difference $\theta$ between the imposed stress and measured strain. Here strain is defined as $\gamma=R \Omega / h$ where $R$ is the radial distance from the spindle axis, $\Omega$ is the angle of displacement and $h$ is the height between the spindle surface and the plate (Fig. 1a). In a cone and plate geometry, $\gamma$ is constant regardless of $R$. Strain rate and phase are obtained from the measured angular velocity and displacement of the spindle. $G^{\prime}$ and $G^{\prime \prime}$ are the storage and loss modulii, respectively and are calculated by the rheometer software. Estimated errors arising from uncertainty in spindle specifications are approximately $0.1,3$, 3\% for stress, strain and modulii respectively. The resolution of strain is approximately $10^{-6}$. We begin each set of measurements at a fixed frequency with a small stress amplitude and incrementally increase the stress amplitude in equal intervals on a log scale. For the measurements at 10 and $1 \mathrm{~Hz}$, the sample is first sheared for 10 cycles at each stress amplitude, and then the measurements are made and averaged over the next 10 cycles. Similarly, for the measurements at $0.1 \mathrm{~Hz}$, the sample is first sheared for 3 cycles and the measurements are made using the average of the next 3-5 cycles. Measurements are done in the order of $10,1,0.1 \mathrm{~Hz}$. We also calculate the internal friction $Q^{-1}$ of the sample using the phase difference as

$Q^{-1}\left(f, \sigma_{0}\right)=\tan \theta=\frac{G^{\prime \prime}}{G^{\prime}}$

Furthermore, we measure the frequency-dependence of the modulii at small stress amplitudes, for which the rheology can be considered to be linearly viscoelastic.

We note that Eqs. ((1)-(2)) are meaningful only for linear viscoelasticity. In the present experiments, non-linear effects become apparent at large amplitudes and the time-dependent strain measurements deviate from a sinusoidal curve. However, similar experiments by Heymann et al. (2002) showed that the amplitudes of the higher modes are less than $15 \%$ of the sinusoidal curve, and we may consider that the same situation applies to our case as well. We also note the flow between the cone and plate can become quite complex and can deviate from ideal shear flow when particle settling occurs (Lenoble et al., 2005). Inhomogeneous particle concentration resulting from shearing can also occur (Huang and Bonn, 2007). For the reasons described above, the modulii obtained should be viewed as effective values assuming linear viscoelasticity and an ideal shear flow, whose effects need to be evaluated in future works.

Particle diameters used in the experiments need to be chosen such that they can be applied to geological situations. Geological suspensions 
are characterized by large particle diameters, typically of the order of $0.1 \mathrm{~mm}$ or larger. Consequently, Brownian motions, which tend to randomize the location of the particles and to homogenize the structural particle network, should be dynamically unimportant. A dimensionless number that compares the particle velocity to the Brownian diffusion velocity is given by the particle Peclet number,

$P e=\frac{6 \pi \eta d^{3} \dot{\gamma}}{k T}$

where $\eta$ is the fluid viscosity, $d$ is the particle diameter, $\dot{\gamma}$ is the strain rate, $k$ is the Boltzmann constant and $T$ is the absolute temperature. The Brownian diffusion velocity is the upper bound, and hence Pe is a lower bound, because in dense suspensions the effective viscosity would be larger. For example, in the case where a seismic wave of $1 \mathrm{~Hz}$ liquefies a water-saturated sand with a particle size of $0.1 \mathrm{~mm}$, the strain amplitude is typically of the order of $10^{-4}$ (Vucetic, 1994). For this case, the Peclet number is $\sim 10^{2}$, and hence Brownian motion is negligible. In our experiments, the corresponding particle Peclet number is also always greater than unity and covers the range $1<P e<10^{11}$. Another key dimensionless parameter is the particle Reynolds number

$R e=\frac{\rho \dot{\gamma} d^{2}}{\eta}$

where $\rho$ is the density. Note again, that our estimate of Re is an upper bound because the effective viscosity increases with packing fraction. At places close to the epicenter of large earthquakes, strain rate can become as large as of the order of $10^{-2} \mathrm{~s}^{-1}$, a situation realized in shear tests of geotechnical engineering (e.g., Hatanaka et al., 1997). When a water-saturated sands with $d \sim 10^{-3} \mathrm{~m}$ is sheared at this strain rate, we have $\operatorname{Re} \sim 10^{-2}$, indicating that particle inertia effects are still small. For a magmatic suspensions, $R e$ is much smaller. The largest $R e$ in our experiments can be evaluated by taking $\dot{\gamma}=1 \mathrm{~s}^{-1}, d=4 \times 10^{-5} \mathrm{~m}$, $\eta=0.95$ Pas from which we obtain $R e \sim 10^{-6}$, indicating that inertial effects are negligible in our experiments as well. (a)

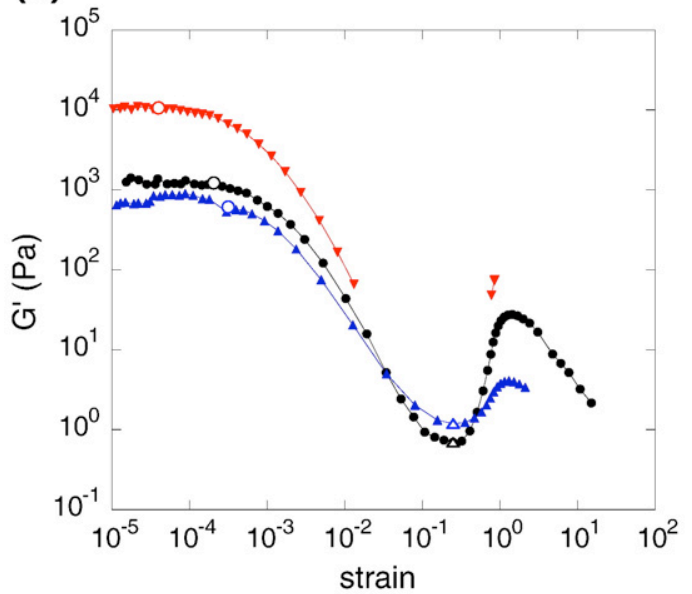

(c)

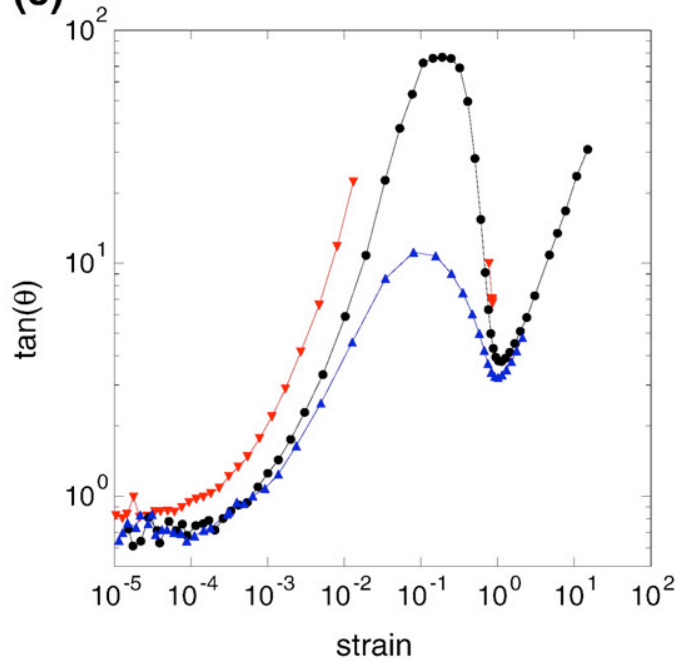

(b)

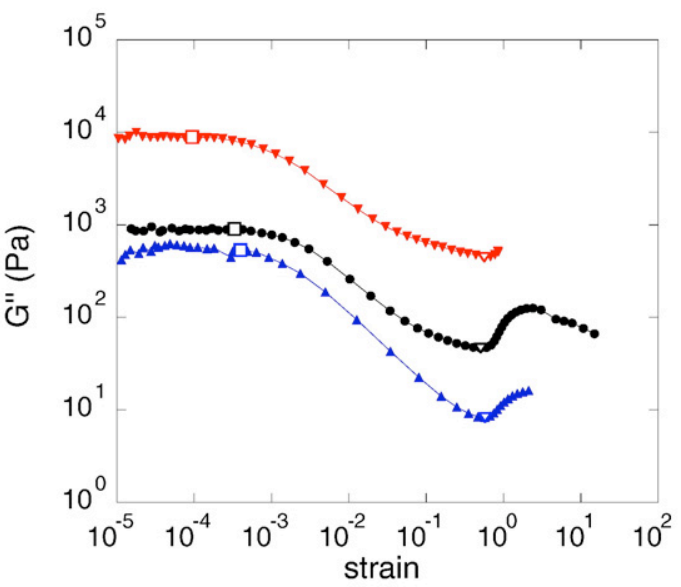

(d)

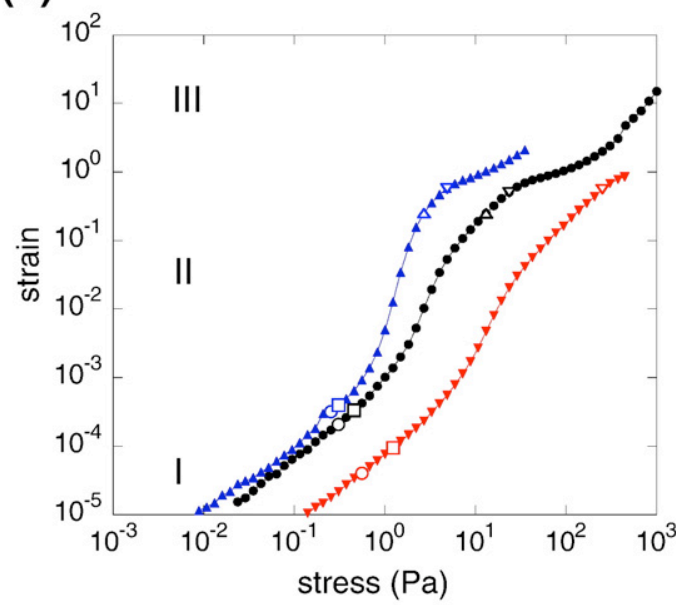

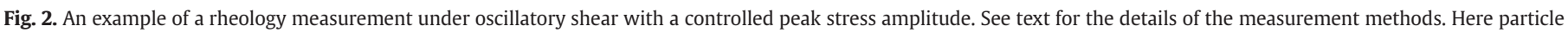

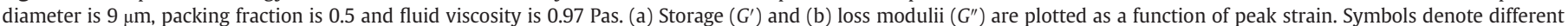

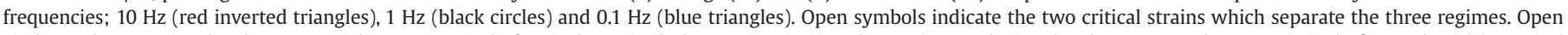

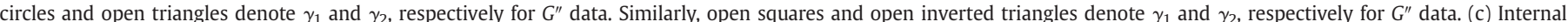

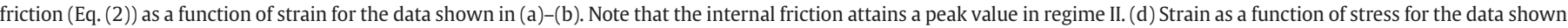

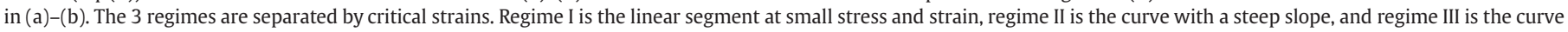
with a smaller slope. (For interpretation of the references to color in this figure legend, the reader is referred to the web version of this article.) 


\section{Results}

\subsection{Rheological regimes under variable-stress-amplitude shear}

Fig. 2 shows typical measurements of the modulii for a suspension with a particle diameter of $9 \mu \mathrm{m}$, packing fraction of 0.5 and a fluid viscosity of 0.97 Pas. Fig. 2(a)-(b) shows the storage and loss modulii as a function of the stress amplitude for 3 different frequencies: 10, 1 and $0.1 \mathrm{~Hz}$. Here we note that although the measurements were made under controlled stress amplitude, we plot measurements as a function of strain because it better defines the different rheological regimes, as we show next.

The measurements show that there are 3 regimes as the stress amplitude is increased. For small stress amplitude, the modulii are independent of peak strain value, and this defines the linear viscoelastic regime (regime I). For intermediate stress amplitude, the modulii show a sharp decline with stress amplitude and this defines the shear-thinning regime (regime II). The decline in storage and loss modulii from the value in regime I to a minimum in regime II are 3 orders and 1 order of magnitude, respectively, indicating that the rheology changes to liquid-like in regime II. For larger stress amplitudes, the modulii recover about a factor of 10 or less and we define this regime as the shear-thickening regime (regime III). Finally for very large stress amplitudes, the modulii decrease again, which we attribute to slip at the interface between the sample and the cone spindle (Larson, 1998), and does not reflect an intrinsic rheological property of the sample.

Fig. 2(c) shows the internal friction (Eq. (2)) as a function of strain for the same measurements. The figure shows that the internal friction attains a maximum in regime II, corresponding to the precipitous decline in storage modulus compared to the loss modulus. This observation indicates that in this regime, a large fraction of the work done to deform the sample is used to transform from solid-like to liquid-like rheology. On the other hand, in regime III, internal friction decreases by regaining strength.

Fig. 2(d) shows the results from the same measurements, plotted as stress versus strain. The three regimes described above are evident as three segments with different slopes. Regime I is the linear segment with a slope of 1 at small stress and strain (linear viscoelastic regime); regime II is the curve with a steeper slope at the intermediate range; regime III is the curve with a smaller slope at large stress and strain.

Fig. 2(a),(b),(d) suggests that strain, rather than stress, better defines the regime II-III transition, and that the strain values are similar regardless of the shearing frequency. We can define the strain

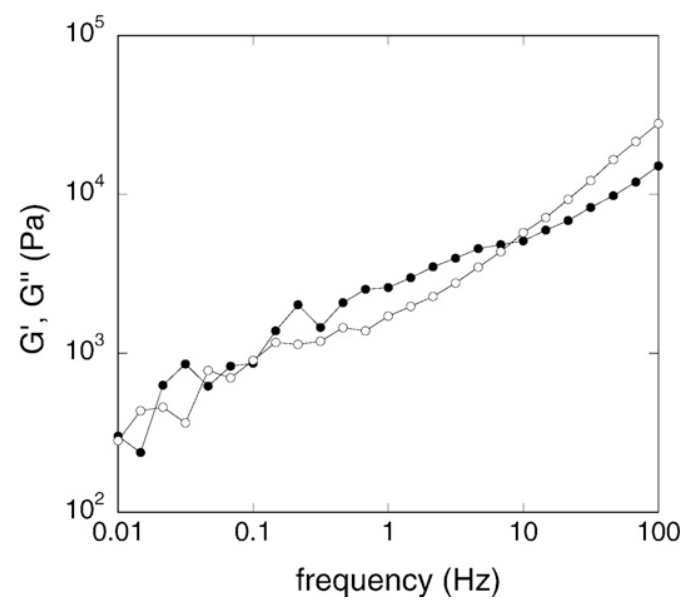

Fig. 3. Frequency-dependence of the modulii in the regime I (linear viscoelastic) for the same sample measured in Fig. 2. Solid and open circles represent $G^{\prime}$ and $G^{\prime \prime}$ respectively. Here a small stress amplitude of $0.1 \mathrm{~Pa}$ was imposed and the oscillatory shear measurement was made by varying the shearing frequency from $0.01 \mathrm{~Hz}$ to $100 \mathrm{~Hz}$. (a)

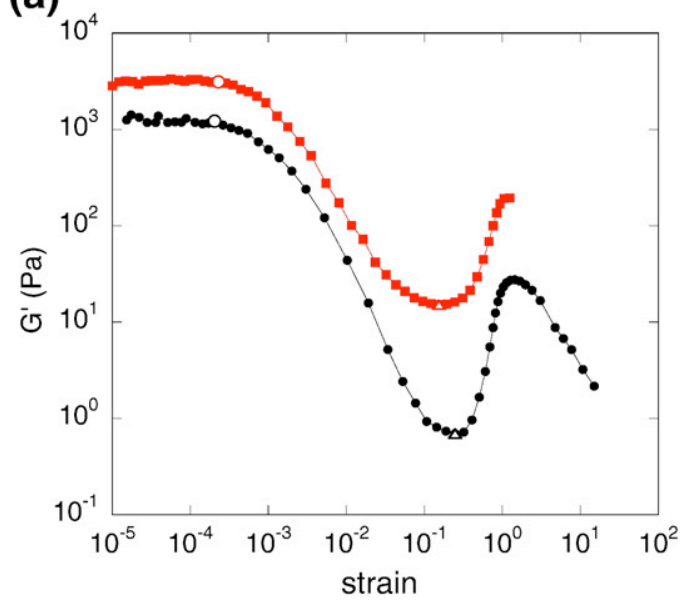

(b)

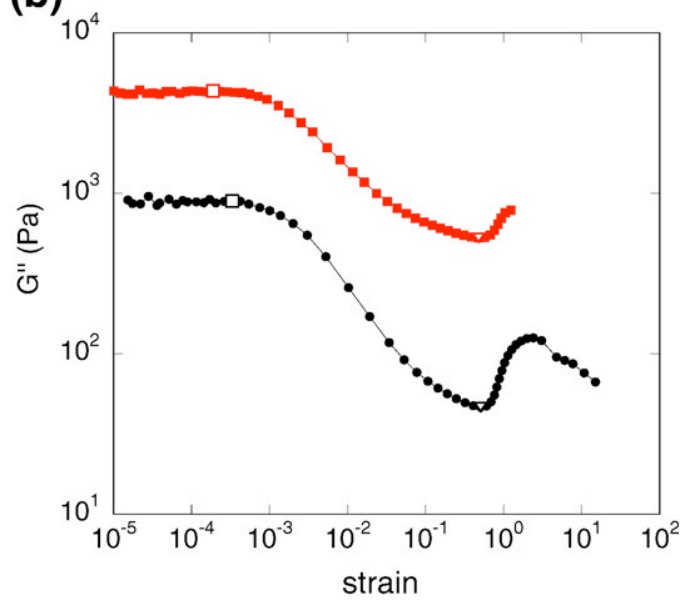

Fig. 4. Viscosity dependence of (a) Storage $\left(G^{\prime}\right)$ and (b) loss modulii $\left(G^{\prime \prime}\right)$ at $1 \mathrm{~Hz}$. Here fluid viscosities are 0.97 Pas (black solid circles) and 12.5 Pas (red solid squares), particle diameter is $9 \mu \mathrm{m}$ and packing fraction is 0.5 . Open symbols indicate the two critical strains which separate the three regimes and are defined in the same way as in Fig. 2. Note that the critical strain values for the two different viscosities are similar. (For interpretation of the references to color in this figure legend, the reader is referred to the web version of this article.)

and stress at which the modulii are minima as the critical strain $\gamma_{2}$ and stress $\sigma_{2}$ that separate regimes II and III. In order to evaluate whether $\gamma_{2}$ or $\sigma_{2}$ better define the regime II-III transition, we compute the relative error (i.e., standard deviation/average) for the $\gamma_{2}$ and $\sigma_{2}$ using the values determined at different frequencies, and find indeed that the error is smaller for $\gamma_{2}$. On the other hand, it is unclear whether strain or stress better defines the regime I-II transition. We define critical strain and stress above which the modulii start to decrease monotonically as $\gamma_{1}$ and $\sigma_{1}$, respectively, and similarly compute the relative error. For the data shown in Fig. 2(d), we find that $\sigma_{1}$ is better for $G^{\prime}$, whereas $\gamma_{1}$ is better for $G^{\prime \prime}$. As we show in Section 3.2, for large packing fractions, we find that $\gamma_{1}$ rather than $\sigma_{1}$ better defines the transition, and choose $\gamma_{1}$ to define the regime I-II transition. The critical strains determined above are indicated in the figures.

Our measurements demonstrate that suspension modulii are frequency-dependent. In regime I, at the same shearing strain or stress amplitude, the modulii are larger for higher frequency. We measured the frequency-dependence of the storage and loss modulii in the linear viscoelastic regime. Results are shown in Fig. 3, and are consistent with the measurements in Fig. 2(a)-(b) at small strains. A crossover of $G^{\prime}$ and $G^{\prime \prime}$ is evident at around 0.1 and $10 \mathrm{~Hz}$, and $G^{\prime}>G^{\prime \prime}$ between these frequencies. This type of frequency-dependence is known to be approximated as a Burgers solid (Findley et al., 1989). 
Similar measurements to those presented in Fig. 2 were made by varying fluid viscosity, packing fraction, and particle diameter. As another example, Fig. 4 shows measured modulii for two different fluid viscosities, 0.97 and 12.14 Pas. Comparing Figs. 2(a)-(b) and 4(a)-(b), we see that the general features characterizing the three regimes are common, and that the values of the critical strains are similar and independent of the fluid viscosity. For this case, we find that critical strain is better than critical stress for both regime I-II and regime II-III transitions.

\subsection{Parameter-dependence of the critical strains}

Here we summarize the results from a series of measurements for variable packing fraction and particle diameter to study how the critical strains depend on these two parameters. Results are shown in Fig. 5(a)-(b). Uncertainties are evaluated from the discretization

\section{(a)}

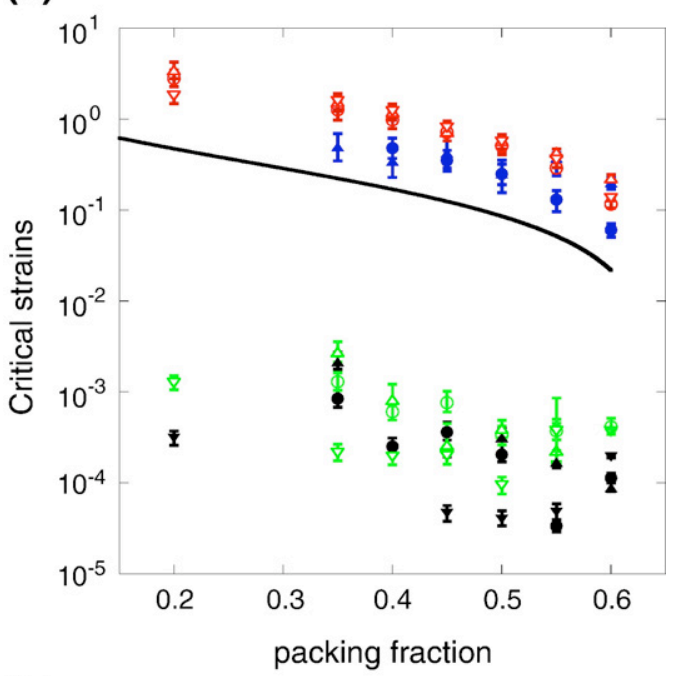

(b)

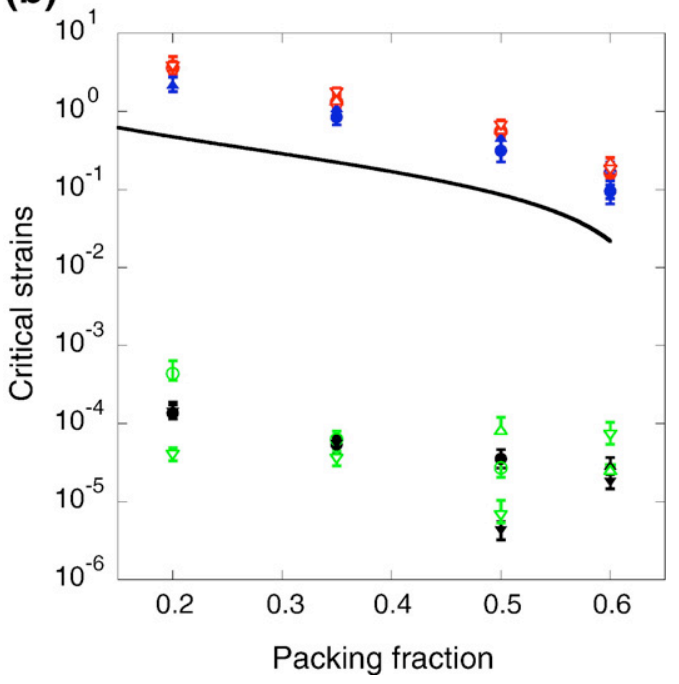

Fig. 5. Critical strains as a function of packing fraction for particle diameters of (a) $9 \mu \mathrm{m}$ and (b) $39 \mu \mathrm{m}$, both suspended in a fluid with a viscosity of 0.97 Pas. Solid symbols are storage modulii $\left(G^{\prime}\right)$ with black and blue indicating $\gamma_{1}$ and $\gamma_{2}$, respectively. Open symbols are loss modulii $\left(G^{\prime \prime}\right)$ with green and red indicating $\gamma_{1}$ and $\gamma_{2}$, respectively. Triangles, circles and inverted triangles are measurements at frequency of $0.1,1$ and $10 \mathrm{~Hz}$, respectively. Error bars are evaluated from the adjacent strain values during the stress sweep. A solid line represents the strain from Bagnold's scaling (Eq. (6)) with a maximum packing fraction of 0.64 . (For interpretation of the references to color in this figure legend, the reader is referred to the web version of this article.)

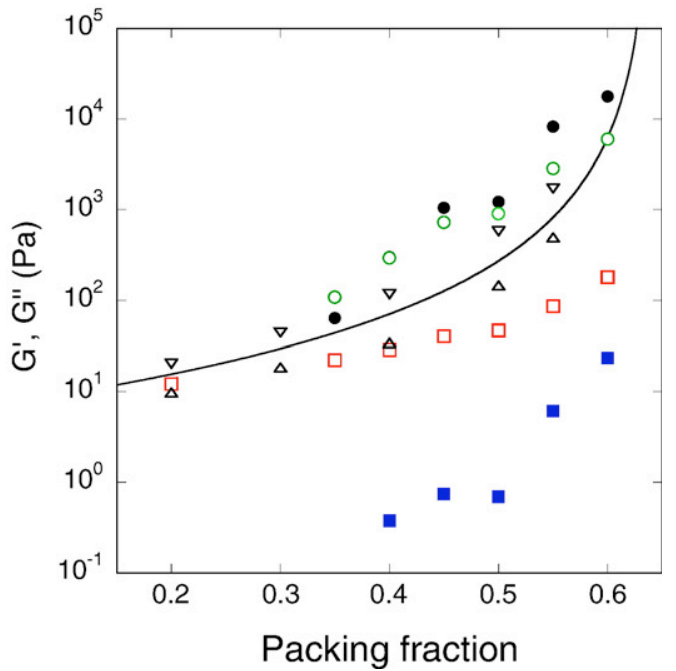

Fig. 6. Storage and loss modulii at $\gamma_{1}$ and $\gamma_{2}$ as a function of packing fraction at frequency of $1 \mathrm{~Hz}$. Here particle diameter is $9 \mu \mathrm{m}$ and the fluid viscosity is 0.97 Pas. Solid symbols are storage modulii $\left(G^{\prime}\right)$ with black circles and blue squares indicating values at $\gamma_{1}$ and $\gamma_{2}$, respectively. Open symbols are loss modulii $\left(G^{\prime \prime}\right)$ with green circles and red squares indicating values at $\gamma_{1}$ and $\gamma_{2}$, respectively. The solid line is the equivalent loss modulii calculated from the Einstein-Roscoe relation with a maximum packing fraction of 0.64 (Eq. (9)). Open inverted triangles and open triangles are the equivalent loss modulii calculated from viscosity measurements of the same sample, using a rotating viscometer at shear rates of 0.1 and $1(1 / \mathrm{s})$, respectively (Michioka, 2005). (For interpretation of the references to color in this figure legend, the reader is referred to the web version of this article.)

of measurements. From Fig. 5, we find that $\gamma_{1}$ is mainly of the order of $10^{-4}$ and $10^{-5}$ for particle diameter of 9 and $39 \mu \mathrm{m}$, respectively, and that there is no clear correlation with the shearing frequency. Though the data are quite scattered, there is a weak trend of decreasing $\gamma_{1}$ with increasing packing fraction. On the other hand, the value of $\gamma_{2}$ is of the order of $10^{-2}$ to 1 and shows a clear decreasing trend with increasing packing fraction. These values are also similar for three different frequencies and two different particle diameters.

Following the method described in Section 3.1, we evaluate whether critical strain or stress better defines the regime transitions using all data plotted in Fig. 5(a)-(b). We find that the regime II-III transition is better defined by strain, rather than stress, for 17 out of 19 cases. On the other hand, for the regime I-II transition, we find that the results depend on the packing fraction. The fractions of cases in which critical strain is better criterion than critical stress are $2 / 7$ for $0.2 \leq \phi \leq 0.45,2 / 4$ for $0.5 \leq \phi \leq 0.55$, and $4 / 4$ for $\phi=0.6$. There is thus an increasing trend of critical strain becoming a better threshold than critical stress as packing fraction increases. To summarize, our experiments suggest that for regime II-III transition, critical strain is a better criterion regardless of packing fraction, whereas for the regime I-II transition, it is better at large packing fractions.

Fig. 6 shows the corresponding modulii at these critical strains. We find that storage and loss modulii are comparable at $\gamma_{1}$, and are increasing functions of packing fraction. On the other hand, at $\gamma_{2}$, $G^{\prime} \ll G^{\prime \prime}$ indicating again that the rheology is liquid-like in regime II.

\subsection{Structural irreversibility and recovery}

In order to better understand the structural changes associated with these different regimes, we made several complementary measurements.

Fig. 7(a) shows how the modulii at a given shearing stress amplitude decrease after imposing a large amplitude shear. First, the imposed stress amplitude was incrementally ramped up to a peak strain slightly above $\gamma_{1}$. Next, the imposed stress amplitude was incrementally ramped down. The trajectory of the measurements indicates that the modulii are similar for the ramp up and down sequences, suggesting that the 
(a)

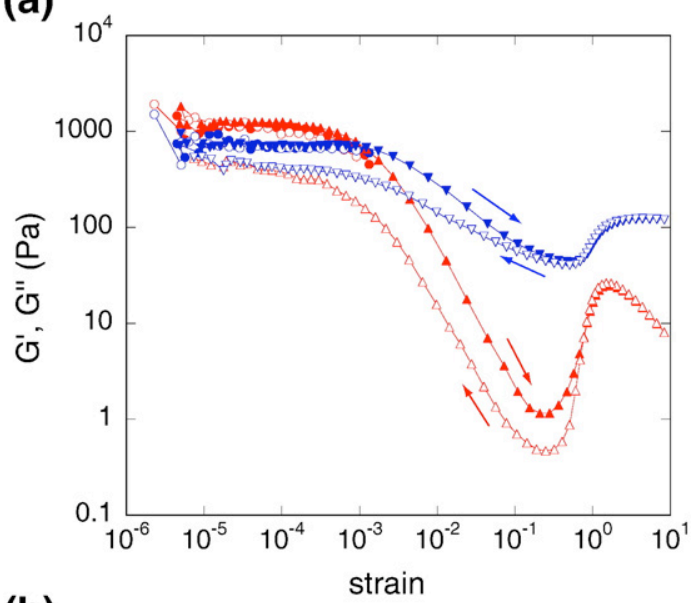

(b)

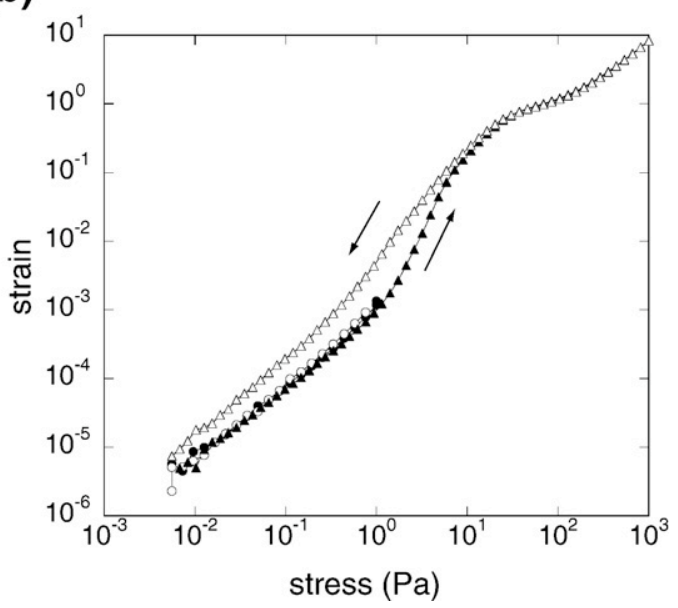

Fig. 7. Rheology measurements at $1 \mathrm{~Hz}$ where the ramp up of the stress amplitude is followed by a ramp down. Here particle diameter is $9 \mu \mathrm{m}$, packing fraction is 0.5 and fluid viscosity is 0.97 Pas. (a) Measurements of storage $\left(G^{\prime}\right)$ and loss modulii $\left(G^{\prime \prime}\right)$, indicated by red and blue symbols, respectively. Solid and open symbols indicate the measurements during ramp up and down, respectively. The first stress loop is indicated by circles. Here, the maximum stress amplitude is $1 \mathrm{~Pa}$ and the resulting maximum strain is $1.338 \times 10^{-3}$. The second stress loop is indicated by triangles for $G^{\prime}$ and inverted triangles for $G^{\prime \prime}$. Here, the maximum stress is $1000 \mathrm{~Pa}$, and the resulting maximum strain is 8.357. Note that the measurements in the first loop overlap (reversible) but in the second loop the modulii are smaller during the ramp down compared to the ramp up (irreversible). (b) Corresponding stress-strain relation. Measurements from the first loop are indicated by solid (ramp up) and open (ramp down) circles. Similarly, measurements from the second loop are indicated by solid and open triangles. An irrecoverable strain is evident after the second loop. (For interpretation of the references to color in this figure legend, the reader is referred to the web version of this article.)

structural changes are small enough that they do not affect the modulii. A new series of measurements was then performed in which the imposed stress amplitude was ramped up again, now to a peak strain greater than $\gamma_{2}$, followed by a similar ramp down. In this sequence, the trajectory of the measurements indicates that the modulii during the ramp down sequence are smaller than the ramp up sequence, notably in the strain range of regimes I and II, indicating that structural changes cause the softening of the sample following the large amplitude oscillatory shear. Fig. 7(b) shows the corresponding stress-strain curve during this sequence showing the presence of an unrecoverable strain after the sample has been sheared above $\gamma_{2}$.

We next monitored the structural recovery after the large amplitude shear was imposed on a suspension with a particle diameter of $9 \mu \mathrm{m}$, whose results are shown in Fig. 8. First, the sample was sheared at a small stress amplitude of 0.1 Pa for $60 \mathrm{~s}$. The measured modulii are shown in Fig. 8(a), and the strain in Fig. 8(b). The strain is of the order of $10^{-5}$ and is in the linear viscoelastic regime. Next, the sample was sheared at a large strain amplitude of 0.1 for $30 \mathrm{~s}$ during which the sample became liquid-like as can be seen from the large decrease in modulii, in particular for the storage modulus. Finally, the sample was sheared again at a small stress amplitude of $0.1 \mathrm{~Pa}$ to monitor the subsequent recovery after the transition to liquid-like behavior in regime II. We find that the modulii recover slowly to the original value, and even after $10^{4}(\mathrm{~s})$ they have not fully recovered. We also conducted the same experiment with a particle diameter of $39 \mu \mathrm{m}$, with other parameters the same and the result of the strain measurement is shown in Fig. 8(b). Comparison shows that recovery for the case of $39 \mu \mathrm{m}$ is faster than the case for $9 \mu \mathrm{m}$.

\section{Discussion of suspension rheology}

\subsection{Comparison with previous rheology measurements}

Here we compare our results with previous related measurements. To our knowledge, previous studies have not defined the critical strains $\gamma_{1}$ and $\gamma_{2}$ in the same manner, nor have they studied the packing

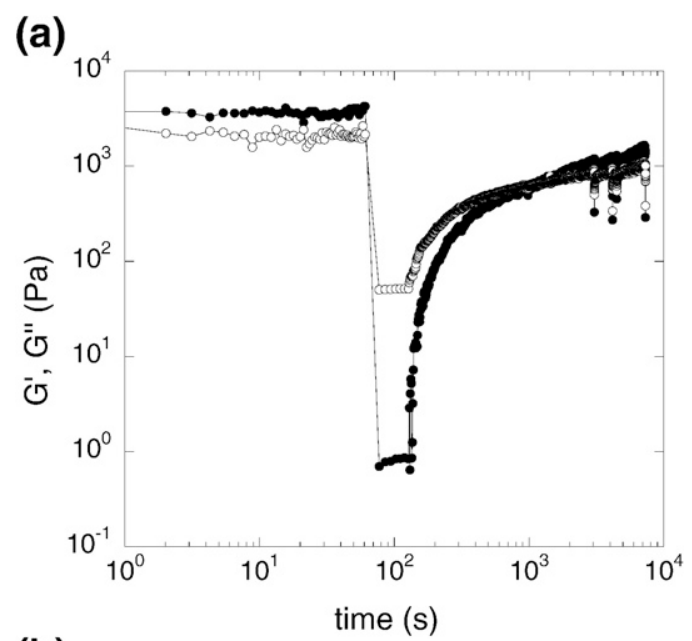

(b)

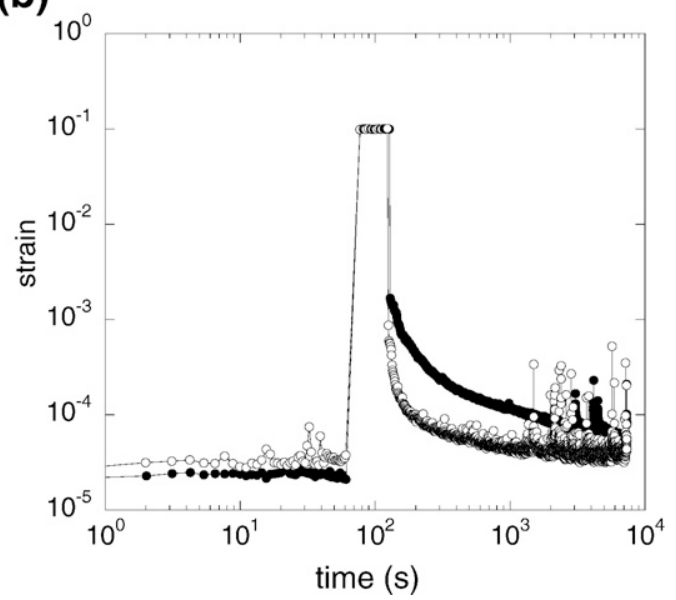

Fig. 8. An experimental run simulating the liquefaction and the subsequent strength recovery of the suspension at frequency of $1 \mathrm{~Hz}$. Here the packing fraction is 0.5 and the fluid viscosity is 0.97 Pas. (a) Temporal change of modulii for $9 \mu \mathrm{m}$ particles. Solid and open circles indicate storage $\left(G^{\prime}\right)$ and loss $\left(G^{\prime \prime}\right)$ modulii, respectively. First, the sample was sheared under a small stress amplitude of $0.1 \mathrm{~Pa}$ for $60 \mathrm{~s}$. Next, it was sheared under a large strain amplitude of 0.1 for $30 \mathrm{~s}$ during which the sample was liquefied. Finally, it was sheared again under a small stress amplitude of 0.1 Pa to monitor the subsequent recovery after liquefaction. (b) Corresponding strain measurements. Solid circles correspond to the strain for the results shown in (a). Open circles correspond to the strain for the results for particle size of $39 \mu \mathrm{m}$, with other parameters the same as those for $9 \mu \mathrm{m}$. 
fraction dependence in the form of plots shown in Fig. 5(a)-(b). Some previous measurements and conclusions, however, are closely related, as we describe below.

First we consider previous oscillatory rheology measurements. The three regimes identified in our measurements have been described previously by Heymann et al. (2002). In their work, the critical strains corresponding to $\gamma_{1}$ and $\gamma_{2}$ were of the order of $10^{-3}$ and $10^{-1}$ respectively, and are similar to our results within an order of magnitude. However, because the frequency range in their measurements was 0.25 $4 \mathrm{~Hz}$, which is more limited compared to ours $(0.1-10 \mathrm{~Hz})$, the stressstrain curves for different frequencies nearly overlapped, i.e., the frequency-dependence of modulii was not evident. As a result, it was unclear whether strain or stress was better suited to define the regime transitions. Importance of strain, rather than stress, on controlling the change in rheology has also been suggested. Narumi et al. (2005) made a similar oscillatory shear measurement for strain amplitudes of up to about 4 , and noticed a distortion of the waveform of the sinusoidal strain curve immediately after the reversal of the direction of shear. The distortion of the strain waveform is independent of shearing frequency, and they defined a characteristic strain of the order of 1 needed for microstructural rearrangement. Comparison with our work suggests that their measurements were made in regime III, and we can infer that this characteristic strain corresponds to the strain needed to initiate the dilatancy. In another example, Pine et al. (2005) tracked the locii of the particles under oscillatory shear and found that above a threshold strain of the order of 1 , the particles do not return to their original positions. This threshold strain was found to decrease with increasing packing fraction, and appears to correspond to $\gamma_{2}$ of our measurements.

Next, we consider cyclic deformation experiments in geotechnical engineering. Here, a threshold cyclic strain has been determined by monitoring pore pressure rise or vertical strain change (Vucetic, 1994; Hsu and Vucetic, 2004, 2006). Typical experiments are done at a shearing frequency of order $0.1 \mathrm{~Hz}$ (e.g., Hsu and Vucetic, 2004). Compilation of results for various sands and soils indicates that threshold strains determined from pore pressure rise and vertical strain are the same, of order $10^{-4}$, and that it is accompanied by a reduction of shear modulus (Vucetic, 1994). Although in our measurements the critical strain threshold is determined in a different way, a good correspondence of the onset of modulus reduction at the threshold strain suggests that our $\gamma_{1}$ corresponds to the threshold strain obtained in geotechnical engineering. Shearing experiments with saturated sands under undrained conditions at larger strains of up to $\gamma \sim 0.1$ have also been made, which showed that stiffening (cyclic mobility) of soil occurs above a strain of the order of $10^{-2}$ (e.g., Hatanaka et al., 1997). This critical strain value is smaller than $\gamma_{2}$ at high packing fractions (0.6) in our experiments by about an order of magnitude or less. Cyclic shearing experiments show that this critical strain asymptotically increases with each cycle, suggesting that a larger critical strain in our measurements may be due to the larger number of such cycles than those in many geotechnical engineering experiments. We can also compare changes in modulii and internal friction. Our experiments documented a 2-4 order of magnitude decrease in the elastic modulus (Fig. 2(a)-(b)), corresponding to a decrease in shear-wave velocity as large as 2 orders of magnitude. Such softening is consistent with previous findings from shear tests in the laboratory (Vucetic, 1994) and in the field (Holzer et al., 1989; Ching and Glaser, 2003; Wang et al., 2006). Our measurements also showed a large increase in internal friction (Eq. (2), Fig. 2(c)), which is also consistent from results in saturated sands (e.g., Kokusho, 1980; Vucetic, 1994).

Finally, we consider unidirectional rheology measurements of dense ( $\phi>0.5)$ suspensions. Ancey (2001) and Huang et al. (2005) found that below a critical shear rate, shear stress is approximately constant (i.e., frictional or viscoelastic) while above this shear rate the shear stress becomes proportional to shear rate (viscous). Huang et al. (2005) concluded that Leighton number, $L e=\frac{\eta \gamma}{\sigma}$ defines the fric- tional-viscous transition, and that there is a critical value $L e_{c} \simeq(7 \pm 5) \times$ $10^{-4}$ above which the flow becomes viscous. Although the style of shearing is different in their experiments compared to ours, we can similarly calculate Le at regime I-II boundary in our experiments by using the maximum shear rate during the oscillatory shear; $\dot{\gamma}=2 \pi f \gamma_{1}$. For the data plotted in Fig. 2, using $\gamma_{1}$ and $\sigma_{1}$ of $G^{\prime}$, we obtain $L e=8 \times 10^{-4}, 4 \times 10^{-3}, 5 \times 10^{-3}$, and using those of $G^{\prime \prime}$, we obtain $L e=8 \times 10^{-4}, 5 \times 10^{-3}, 5 \times 10^{-3}$, for the shearing frequency of $0.1,1$, $10 \mathrm{~Hz}$, respectively. Le for different frequencies are similar within an order of magnitude because $\sigma$ increases with $\dot{\gamma}$. Our calculated $L e$ are comparable to $L e_{c}$ of Huang et al. (2005) within a factor of 5, indicating that $\gamma_{1}$ may be interpreted as a frictional-viscous transition. We can also compare the packing fraction dependence which has been studied by Watanabe et al. (1999), where they imposed a steady shear to a suspension and monitored how the viscosity increased with time to reach a steady-state. They found that when the imposed strain exceeded a critical value of order $10^{-1}$, the viscosity approached a terminal value, and that this critical strain decreases with packing fraction. Although the style of measurement is different, the critical strain is of the same order of magnitude as $\gamma_{2}$, and suggests a common mechanism. As another example, Uhlherr et al. (2005) made similar measurements and showed that yielding occurs when the accumulated strain reaches a critical value of around 1, and suggested using yield strain to characterize the threshold for flowing, thus consistent with our results.

\subsection{Origin of the critical strains}

First we consider $\gamma_{2}$, the critical strain at which shear-thinning transforms to shear-thickening. We infer from this transition that the particle alignments favorable for deformation are destroyed above this strain and, as a result, the strength recovers. One measure of such critical strain was provided by Bagnold (1954) as

$\gamma=\frac{\delta}{d}$

which is the strain needed for the adjacent particles to collide. Here, $\delta$ is the distance between the adjacent particles and $d$ is the particle diameter. Note that this is a geometrical relation, not limited to linear viscoelasticity. An essentially identical expression has been used in Watanabe et al. (1999). Because $\phi \propto(d+\delta)^{-3}$ and $\phi_{\text {max }} \propto d^{-3}$ where $\phi$ and $\phi_{\text {max }}$ are packing fraction and its maximum value, respectively, we obtain

$\gamma=\left(\frac{\phi}{\phi_{\max }}\right)^{-1 / 3}-1$

For random close packing $\phi_{\max } \simeq 0.64$ (Mavko et al., 1998): using this value, we plot Eq. (6) in Fig. 5(a)-(b). Comparison with our measurements indicate that although the model is smaller by about a factor of 5 , it captures the decreasing trend well.

Next, we consider $\gamma_{1}$, the critical strain above which the change in microstructure starts to cause the decrease of the modulii. In dense suspensions, particles are in contact with adjacent particles and the suspension is in a jammed state. When the suspension is sheared at small strains $<\gamma_{1}$, the contacting adjacent particles slip, friction is reduced, and the suspension eventually unjams as contacts are loosened. We do not have a quantitative theory for $\gamma_{1}$, but we can hypothesize as follows. First we evaluate the magnitude of the various forces which act on a particle with a diameter of $9 \mu \mathrm{m}$. During shearing, the viscous force imposed on a particle is $>3 \times 10^{-12} \mathrm{~N}$. On the other hand, the negative buoyancy force is $\simeq 3 \times 10^{-13} \mathrm{~N}$, the inertial force is $\sim(4 / 3) \rho(d / 2)^{4} \gamma^{2}<2 \times 10^{-16} \mathrm{~N}$, and the van der Waals attractive force can be estimated as (Larson, 1998)

$F_{\mathrm{vdw}}=\frac{A_{H} d}{12 \delta^{2}}$ 
where $A_{H}$ is the Hamaker parameter, $\delta$ is the gap between the particles. Here $A_{H}$ depends on distance and becomes very small for $\delta>5-10 \mathrm{~nm}$ (Larson, 1998), and thus the attractive force becomes negligibly small. $A_{H}$ can be estimated from surface tension (Larson, 1998), and for polystyrene-silicone oil we obtain $A_{H} \sim 8 \times 10^{-21} \mathrm{~J}$. Here we used $43 \mathrm{mN} / \mathrm{m}$ (Hata et al., 1987) and $21 \mathrm{mN} / \mathrm{m}$ for the surface tension of polystyrene and silicone oil, respectively. The mean value of $\delta$ decreases with packing fraction. Using the expression

$\delta=d\left(\left(\frac{1}{3 \pi \phi}+\frac{5}{6}\right)^{1 / 2}-1\right)$

(Woodcock, 1987) we infer $\delta \geq 45 \mathrm{~nm}$, where the minimum value is for the case with $\phi=0.6$, and thus obtain $F_{\mathrm{vdw}}<3 \times 10^{-12} \mathrm{~N}$ at the mean particle distance. This estimate shows that for even the smallest applied stress, viscous forces are comparable to or larger than the van der Waals forces, and that they always overwhelm buoyancy and inertial forces, indicating that the primary force balance is between viscous and pressure forces. van der Waals force can, however become important at points of near particle contact $(\delta<10 \mathrm{~nm})$. The critical strain of $\gamma_{1} \sim 10^{-4}$, when scaled to the particle diameter of $d=9 \mu \mathrm{m}$, corresponds to a displacement of a few $\mathrm{nm}$, and therefore may be related to the distance needed to overcome attractive forces. Our experiments also indicate that critical strain is smaller for larger particle size $\left(\gamma_{1} \sim 10^{-5}\right.$ for $\left.d=39 \mu \mathrm{m}\right)$, which is consistent with the existence of such critical displacement scale for unjamming.

\subsection{Modulii values at critical strains}

Fig. 6 shows that at both $\gamma_{1}$ and $\gamma_{2}, G^{\prime} / G^{\prime \prime}$ increases with packing fraction, demonstrating the increasing solid-like behavior with packing fraction. We compare our measurements with an empirical Einstein-Roscoe formula e.g., (McBirney and Murase, 1984) or equivalently a Krieger-Dougherty formula (Krieger and Dougherty, 1959), which provides a relationship between the packing fraction and viscosity. We calculate the equivalent loss modulus as

$G^{\prime \prime}=2 \pi f \eta\left(1-\frac{\phi}{\phi_{\max }}\right)^{-2.5}$

and plot Eq. (9) in Fig. 6. Here we take $f=1 \mathrm{~Hz}, \eta=0.97$ Pas. In this figure, we also plot the equivalent loss modulii calculated from viscosity values at shear rates of 0.1 and $1 \mathrm{~s}^{-1}$ for the same sample measured using a rotating viscometer (Brookfield RVDV2+PRO) in a vane-in-cup geometry (Michioka, 2005). Here, we changed the spindle rotation rate from 0.01 to $10 \mathrm{rpm}$, and calculated the shear rate using the method described in Spera et al. (1988) to obtain the viscosity. From Fig. 6, we find that the equivalent loss modulii values are intermediate of the $G^{\prime \prime}$ values at $\gamma_{1}$ and $\gamma_{2}$. Viscosity is measured under a unidirectional shear which corresponds to an infinite amplitude limit of oscillatory shear, and is similar to regime III in our experiments. Modulii values in regime III are in between those at $\gamma_{1}$ and $\gamma_{2}$ and are therefore consistent. We note that in the measurements using the rotating viscometer, viscosity is shear-thinning up to the largest shear rate $\left(\sim 2 \mathrm{~s}^{-1}\right)$ attained, and that no shear-thickening was observed. Comparing with the results reported in this paper, we suggest that the reversal of shearing direction promotes shear-thickening.

We can also compare our result with the modified Cox-Merz rule for concentrated suspensions (Gleissle and Hochstein, 2003). This rule states $\left|\eta^{*}(\omega)\right|=\eta(\phi)$ which can be rewritten as $\left|G^{*}(\omega)\right|=\omega \eta(\phi)$. Here $\left|\eta^{*}(\omega)\right|$ is the magnitude of the complex viscosity at angular frequency $\omega$, and $\eta(\phi)$ is the viscosity at packing fraction $\phi$. In our measurements at $\gamma_{1}$, we find that $G^{\prime}(\omega) \simeq G^{\prime \prime}(\omega)$ from which we have $\left|G^{*}(\omega)\right| \simeq \sqrt{2}\left|G^{\prime}(\omega)\right|$. Also at $\gamma_{2}$, we have an approximate relation $\left|G^{*}(\omega)\right| \simeq\left|G^{\prime \prime}(\omega)\right| \gg\left|G^{\prime}(\omega)\right|$. Fig. 6, together with these approximations indicates that $\left|G^{*}(\omega)_{\gamma=\gamma_{2}}\right|<$ $\omega \eta(\phi)|<| G^{*}(\omega)_{\gamma=\gamma_{1}} \mid$, which shows that complex modulus estimated using the modified Cox-Merz rule and the viscosity values that are measured and also estimated from Einstein-Roscoe's relation are bounded by complex modulii at $\gamma_{1}$ and $\gamma_{2}$.

\subsection{Scaling of the recovery time scale}

We next consider the cause for the faster recovery for larger particle size as observed in Fig. 8(b). One possible explanation is that the recovery is due to the relaxation of the anomalous pore pressure driven by viscous permeable flow, which is plausible from the force balance arguments in Section 4.2. Liquefaction occurs when the anomalous pore pressure builds up to the point that it can support the dispersed particles. This pressure relaxes by permeable flow when the large amplitude oscillatory shear is removed and can be described by a diffusion equation with a characteristic time scale

$$
\tau \sim \frac{h^{2} \eta}{k_{\phi} E}
$$

where $h$ is the thickness of the sample, $\eta$ is the fluid viscosity, $k_{\phi}$ is the permeability and $E$ is the composite stiffness modulus which can be approximated by $E \sim G^{\prime}$. Here we assumed that the vertical pressure gradient $\gg$ horizontal pressure gradient. This approximate expression gives only an order of magnitude estimate but has been used in estimating pore pressure decay time scale (Iverson, 1997) as well as the response time of stressed gels (Ketz et al., 1988). For permeability, we use the Blake-Kozeny-Carman formula

$k_{\phi}=\frac{d^{2}}{K} \frac{(1-\phi)^{2}}{\phi^{3}}$,

where $K$ is an empirical constant, $\sim 180$ (Bear, 1972). In the present case, as recovery proceeds $E$ increases and $k_{\phi}$ decreases with time. As a crude approximation we treat the denominator of Eq. (10) as invariant. For the measurement shown in Fig. 8(b), $\eta=0.97$ Pas, $\phi=0.5$, and we use $h=2.1 \times 10^{-3} \mathrm{~m}$ for the sample thickness at the edge of the cone spindle. For the case of $9 \mu \mathrm{m}$ particles, we take $G^{\prime} \sim 10^{2} \mathrm{~Pa}$ (Fig. 8 (a)), to obtain $\tau \sim 2 \times 10^{5} \mathrm{~s}$. Our measurements were not made over a long enough time period to observe total recovery, but extrapolation suggests that our estimate is consistent with the measurements. For the case of $d=39 \mu \mathrm{m}$, we take $G^{\prime} \sim 10^{2} \mathrm{~Pa}$ (data not shown) to obtain $\tau \sim 1 \times 10^{4} \mathrm{~s}$, in approximate agreement with the strain relaxation time scale of $\sim 10^{4} \mathrm{~s}$ shown in Fig. 8(b). A similar slow recovery (thixotropy) after the suspension has been deformed is also demonstrated in other studies (Völtz et al., 2002; Stokes et al., 2005).

\section{Geophysical implications}

First, we consider the possibility of initiating volcanic activity or eruption through the seismically-induced enhanced mobility or liquefaction of a crystal-rich viscous magma, as proposed by Hill et al. (2002). Magma can allow shear waves to propagate if it is sheared at a period shorter than the Maxwell relaxation time $\eta / G$, where $\eta$ is the viscosity and $G$ is the shear modulus. Viscosity of silicate liquid is strongly temperature dependent, and the relaxation time increases with cooling because $\eta$ is very sensitive to temperature compared to $G$ (Webb, 1997). Below the liquidus, temperature dependent viscosity and crystal content both affect the relaxation time. Measurements for partiallymolten granites (Bagdassarov and Dorfman, 1998) indicate that relaxation time increases further when cooled below liquidus, such that propagation of shear waves becomes possible at seismic frequencies of $\sim 1 \mathrm{~Hz}$. We also note because of the impedance contrast of the magma chamber relative to that of the country rock, an amplification of shear wave propagating into the magma chamber is possible, though because partially-molten rock has high attenuation, shear waves may also attenuate rapidly. We thus conclude that the mobility of crystal-rich magma can be enhanced provided that the strain amplitude $>\gamma_{1} \sim 10^{-4}$. 
Earthquake-triggered volcanic eruptions in which eruption begins within days of large, distant earthquakes, has been analyzed by Linde and Sacks (1998) and redone by Manga and Brodsky (2006) using updated earthquake and eruption catalogues. In Fig. 9 we plot the relationship between earthquake magnitude and the distance from the earthquake for these triggered eruptions from the data given in Table 1 of Manga and Brodsky (2006). For comparison, Fig. 9 also shows hydrologic responses to earthquakes, in particular, increases in stream flow, the eruption of mud volcanoes, and the occurrence of nearsurface liquefaction. The source of data for the hydrologic responses are from compilations in Montgomery and Manga (2003), Mellors et al. (2007) and Manga (2007). The solid line is an empirically determined upper bound for the distance over which most of these hydrologic responses occur (Wang et al., 2006). Fig. 9 shows that triggered volcanic eruptions generally fall within a magnitude-distance bound similar to that for hydrologic responses. This correlation suggests a common mechanism, and hence the possibility that liquefaction may play a role in triggering volcanic eruptions. Seismic magnitude is related to seismic-wave energy density $\sim(1 / 2) G \gamma^{2}$ where $G$ is the effective shear modulus and $\gamma$ is the strain amplitude. The shear modulus of both water-saturated sands (Kokusho, 1980) and basalts near melting point (James et al., 2004) at seismic frequencies is of the order of $100 \mathrm{MPa}$. Therefore, the similarity of the liquefaction limits for hydrologic and magma is consistent with similarity of values of shear modulii, assuming that the critical strains are independent of viscosity values as verified in our experiments.

Although not all hydrologic responses are caused by liquefaction (Rojstaczer et al., 1995; Wang et al., 2004), all these hydrologic responses are associated with increases in fluid pressure and permanent changes in structure. Manga et al. (2003) showed that seismic strain amplitudes that cause increases in stream flow are $O\left(10^{-4}\right)$. This is the same order of magnitude as the strains that permit structural

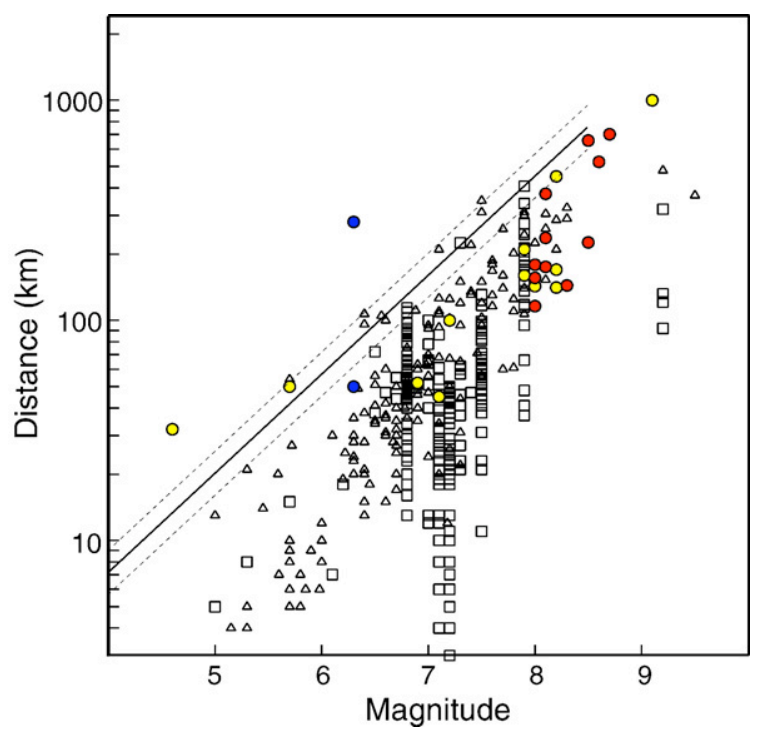

Fig. 9. Distance between the location of volcanic or hydrologic responses to earthquakes and the epicenter of the earthquakes, as a function of the earthquake magnitude. The volcanic eruptions shown with red circles are listed in Table 1 of Manga and Brodsky (2006) and all erupted within 5 days of the earthquake. Only earthquakes with magnitudes greater than 8 were considered. The blue circles show volcanoes that were already erupting and exhibited an increase in eruption rate (Harris and Ripepe, 2007). The yellow circles show triggered eruptions of mud volcanoes (Manga and Brodsky, 2006; Mellors et al., 2007). The open triangles are occurrences of (shallow) liquefaction and the open squares are locations with increases in stream flow; sources of data and references are from Montgomery and Manga (2003), Wang et al. (2006), Manga (2007). The solid line is a threshold of the best-fit liquefaction limit from Wang et al. (2006), with the dashed lines showing the standard error calculated for this threshold. (For interpretation of the references to color in this figure legend, the reader is referred to the web version of this article.) changes and result in liquid-like behavior in our experiments. We expect similar strain amplitudes for the triggered eruptions shown in Fig. 9, though the actual values depend on the geometry of the magma bodies and elastic modulii of the crystal-rich magma. We also note that our measurements showed that shear modulus is an increasing function of fluid viscosity. If we assume that the threshold strain is independent of viscosity, it follows that a more viscous magma may require a larger magnitude or a smaller epicentral distance to trigger eruption.

Second, we consider the possibility of triggering of earthquakes by seismic waves in geothermal areas. Such examples are reported in Long Valley caldera and the Geysers where local earthquakes occurred during the passage of surface waves (Hill et al., 1993). Brodsky and Prejean (2005) analyzed remotely triggered seismicity at Long Valley caldera and found that for seismic waves with comparable velocity amplitude, longer period waves are more effective in generating local seismicity. Their observation is consistent with our finding that strain governs the rheology, because under the same velocity amplitude, longer period waves have larger displacement (and hence strain) amplitudes. Triggered earthquakes in geothermal areas can also, in some cases, continue long after the propagation of surface waves (e.g., Brodsky, 2006). Our experiments show that strain relaxation can be approximately scaled as Eq. (10) which is proportional to fluid viscosity. This indicates that viscous magmas have long stress relaxation times and can cause prolonged periods of triggered earthquakes. Our measurements also showed that shear-wave modulii (and hence velocity) and attenuation depend strongly on amplitude (Fig. 2(a)-(c)), and that such properties may need to be considered for near-field wave propagation. This implies that a volcano or a magma chamber may act as an amplitude-selective filter of seismic waves, and that triggered eruption is most effective for a certain amplitude band corresponding to regime II.

\section{Acknowledgements}

We thank Y. Guéguen, an anonymous referee, and the editor for helpful comments on the manuscript. The measurements were done at University of California, Berkeley during which I.S. was supported by MEXT Japan. We thank A. Namiki, H. Nakamichi, C. Wang, J. Dufek for their help and discussions. This work was supported by JSPS (I.S.) and NSF EAR-0608885 (M.M.).

\section{References}

Ancey, C., 2001. Role of lubricated contacts in concentrated polydisperse suspensions. J. Rheol. 45, 1421-1439.

Bagdassarov, N., Dingwell, D.B., Webb, S.L., 1994. Viscoelasticity of crystal- and bubble bearing rhyolite melts. Phys. Earth Planet. Inter. 83, 83-99.

Bagdassarov, N., Dorfman, A., 1998. Viscoelastic behaviour of partially molten granites. Tectonophys 290, 27-45.

Bagnold, R.A., 1954. Experiments on a gravity-free dispersion of large solid spheres in a Newtonian fluid under shear. Proc. R Soc. Lond. Ser. A. 225, 49-63.

Bear, J., 1972. Dynamics of fluids in porous media. American Elsevier, New York.

Brodsky, E.E., 2006. Long-range triggered earthquakes that continue after the wave train passes. Geophys. Res. Lett. 33. doi:10.1029/2006GL026605.

Brodsky, E.E., Prejean, S.G., 2005. New constraints on mechanisms of remotely triggered seismicity at Long Valley Caldera. J. Geophys. Res. 110. doi:10.1029/2004JB003211.

Ching, J., Glaser, S., 2003. Identification of soil degradation during earthquake excitations by Bayesian inference. Earthquake Eng. Struct. Dyn. 32, 845-869.

Findley, W.N., Lai, J.S., Onaran, K., 1989. Creep and relaxation of nonlinear viscoelastic materials: with an introduction to linear viscoelasticity. Dover Publications.

Fontaine, F.R., Ildefonse, B., Bagdassarov, N.S., 2005. Temperature dependence of shear wave attenuation in partially molten gabbronorite at seismic frequencies. Geophys. J. Int. 163, 1025-1038.

Gleissle, W., Hochstein, B., 2003. Validity of the Cox-Merz rule for concentrated suspensions. J. Rheol. 47, 897-910.

Harris, A.J.L., Ripepe, M., 2007. Regional earthquake as a trigger for enhanced volcanic activity: evidence from MODIS thermal data. Geophys. Res. Lett. 34. doi:10.1029/ 2006GL028251.

Hata, T., Kitazaki, Y., Saito, T., 1987. Estimation of the surface energy of polymer solids. J. Adhes. 21, 177-194.

Hatanaka, M., Uchida, A., Ohara, J., 1997. Liquefaction characteristics of a gravelly fill liquefied during the 1995 Hyogo-ken Nanbu earthquake. Soil Found. 37, 107-115. 
Heymann, L., Peukert, S., Aksel, N., 2002. Investigation of the solid-liquid transition of highly concentrated suspensions in oscillatory amplitude sweeps. J. Rheol. 46, 93-112.

Hill, D.P., Reasenberg, P.A., Michael, A., Arabaz, W.J., Beroza, G., Brumbaugh, D., Brune, J.N., Castro, R., Davis, S., dePolo, D., Ellsworth, W.L., Gomberg, J., Harmsen, S., House, L. Jackson, S.M., Johnston, M.J.S., Jones, L., Keller, R., Malone, S., Munguia, L., Nava, S., Pechmann, J.C., Sanford, A., Simpson, R.W., Smith, R.B., Stark, M., Stickney, M., Vidal, A., Walter, S., Wong, V., Zollweg, J., 1993. Seismicity remotely triggered by the magnitude 7.3 Landers, California, Earthquake. Science 260, 1617-1623.

Hill, D.P., Pollitz, F., Newhall, C., 2002. Earthquake-volcano interactions. Phys. Today 55, 41-47.

Holzer, T.L., Tinsley, J.C., Hank, T.C., 1989. Dynamics of liquefaction during the 1987 Superstition Hills, California, earthquake. Science 244, 56-59.

Hsu, C.-C., Vucetic, M., 2004. Volumetric threshold shear strain for cyclic settlement. J. Geotech. Geoenviron. Eng. 130, 58-70.

Hsu, C.-C., Vucetic, M., 2006. Threshold shear strain for cyclic pore-water pressure in cohesive soils. J. Geotech. Geoenviron. Eng. 132, 1325-1335.

Huang, N., Bonn, D., 2007. Viscosity of a dense suspension in Couette flow. J. Fluid Mech. 590, 497-507.

Huang, N., Ovarlez, G., Bertrand, F., Rodts, S., Coussot, P., Bonn, D., 2005. Flow of wet granular materials. Phys. Rev. Lett. 94, 028301

Iverson, R.M., 1997. The physics of debris flows. Rev. Geophys. 35, 245-296.

James, M.R., Bagdassarov, N., Müller, K., Pinkerton, H., 2004. Viscoelastic behaviour of basaltic melts. J. Volcanol. Geotherm. Res. 132, 99-113.

Ketz Jr., R.J., Prud'homme, R.K., Graessley, W.W., 1988. Rheology of concentrated microgel solutions. Rheol. Acta 27, 531-539.

Koenders, M.A., Petford, N., 2000. Quantitative analysis and scaling of sheared granitic magmas. Geophys. Res. Lett. 27 (8), 1231-1234.

Kokusho, T., 1980. Cyclic triaxial test of dynamic soil properties for wide strain range. Soil Found. 20, 45-60.

Krieger, I.M., Dougherty, T.J., 1959. A mechanism for non-Newtonian flow in suspensions of rigid spheres. Trans. Soc. Rheol. 3, 137-152.

Larson, R.G., 1998. The structure and rheology of complex fluids. Oxford Univ. Press. $663 \mathrm{pp}$.

Lenoble, M., Snabre, P., Pouligny, B., 2005. The flow of a very concentrated slurry in a parallel-plate device: influence of gravity. Phys. Fluids 17, 073303.

Linde, A.T., Sacks, I., 1998. Triggering of volcanic eruptions. Nature 395, 888-890.

Manga, M., 2007. Did an earthquake trigger the May 2006 eruption of the Lusi mud volcano? EOS 88, 201.

Manga, M., Brodsky, E., 2006. Seismic triggering of eruptions in the far field: volcanoes and geysers. Ann. Rev. Earth Planet. Sci. 34, 263-291.

Manga, M., Brodsky, E.E., Boone, M., 2003. Response of stream flow to multiple earthquakes. Geophys. Res. Lett. 30. doi:10.1029/2002GL016618.

Mavko, G., Mukerji, T., Dvorkin, J., 1998. The Rock Physics Handbook. Cambridge University Press, p. 329.
McBirney, A.R., Murase, T., 1984. Rheological properties of magmas. Ann. Rev. Earth Planet. Sci. 12, 337-357.

Mellors, R., Kilb, D., Aliyeev, A., Gasanov, A., Yetirmishli, G., 2007. Correlations between earthquakes and large mud volcano eruptions. J. Geophys. Res. 112, B04304. doi:10.1029/2006JB004489.

Michioka, H., 2005. Rayleigh-Taylor instability and rheology of a particle packed viscous fluid. Master Thesis, Kanazawa University.

Montgomery, D.R., Manga, M., 2003. Streamflow and water well responses to earthquakes. Science 300, 2047-2049.

Narumi, T., See, H., Suzuki, A., Hasegawa, T., 2005. Response of concentrated suspensions under large amplitude oscillatory shear flow. J. Rheol. 49 (1), 71-85.

Petford, N., Koenders, M.A., 2003. Shear-induced pressure changes and seepage phenomena in a deforming porous layer - i. Geophys. J. Int. 155, 857-869.

Pine, D.J., Gollub, J.P., Brady, J.F., Leshansky, A.M., 2005. Chaos and threshold for irreversibility in sheared suspensions. Nature 438, 997-1000.

Rojstaczer, S., Wolf, S., Michel, R., 1995. Permeability enhancement in the shallow crust as a cause of earthquake-induced hydrological changes. Nature 373, 237-239.

Spera, F.J., Borgia, A., Strimple, J., 1988. Rheology of melts and magmatic suspensions 1. design and calibration of concentric cylinder viscometer with application to rhyolitic magma. J. Geophys. Res. 93, 273-294.

Stokes, J.R., Telford, J.H., Williamson, A.-M., 2005. The flowability of ice suspensions. J. Rheol. 49 (1), 139-148.

Uhlherr, P.H.T., Guo, J., Tiu, C., Zhang, X.-M., Zhou, J.Z.Q., Fang, T.-N., 2005. The shearinduced solid-liquid transition in yield stress materials with chemically different structures. J. Non-Newton. Fluid Mech. 125, 101-119.

Völtz, C., Nitschke, M., Heymann, L., Rehberg, I., 2002. Thixotropy in macroscopic suspensions of spheres. Phys. Rev., E 65, 051402.

Vucetic, M., 1994. Cyclic threshold shear strains in soils. J. Geotech. Eng. 120 (12) 2208-2228.

Wang, C.-Y., Wang, C.-H., Manga, M., 2004. Coseismic release of water from mountains: evidence from the $1999\left(M_{w}=7.5\right)$ Chi-Chi Taiwan earthquake. Geology 32, 769-772.

Wang, C.-Y., Wong, A., Dreger, D.S., Manga, M., 2006. Liquefaction limit during earthquakes and underground explosions: implications for ground-motion attenuation. Bull. Seismol. Soc. Am. 96, 355-363.

Watanabe, H., Yao, M.-L., Osaki, K., Shikata, T., Niwa, H., Morishima, Y., 1999. Nonlinear rheology of concentrated spherical silica suspensions: 3. concentration dependence. Rheol. Acta 38, 2-13.

Webb, S., 1997. Silicate melts: relaxation, rheology, and the glass transition. Rev Geophys. 35, 191-219.

Woodcock, L.V., 1987. Molecular dynamics and relaxation phenomena in glasses. Proc Workshop held at the Zentrum für Interdisziplinäre, vol. 277. Forschung Universitüat Bielfeld, pp. 113-124. 